\title{
Interdomain electron transfer in cellobiose dehydrogenase is governed by surface electrostatics
}

Alan Kadek ${ }^{\mathrm{a}, \mathrm{b}}$, Daniel Kavan ${ }^{\mathrm{a}, \mathrm{b}}$, Julien Marcoux ${ }^{\mathrm{c}, \mathrm{d}, \#, \text { Johann Stojko }}{ }^{\mathrm{c}, \mathrm{d}}$, Alfons K.G. Felice ${ }^{\mathrm{e}}$, Sarah Cianféranic ${ }^{c, d}$, Roland Ludwig ${ }^{\mathrm{e}}$, Petr Halada ${ }^{\mathrm{a}}$, Petr Man ${ }^{\mathrm{a}, \mathrm{b}, *}$

${ }^{a}$ BioCeV - Institute of Microbiology, The Czech Academy of Sciences, Prumyslova 595, Vestec, Czech Republic

${ }^{b}$ Department of Biochemistry, Faculty of Science, Charles University in Prague, Hlavova 8 , Prague, Czech Republic

${ }^{c}$ BioOrganic Mass Spectrometry Laboratory (LSMBO), IPHC, Université de Strasbourg, 25 rue Becquerel, 67087 Strasbourg, France

${ }^{d}$ IPHC, CNRS, UMR7178, 67087 Strasbourg, France

${ }^{e}$ Department of Food Sciences and Technology, BOKU - University of Natural Resources and Life Sciences, Muthgasse 18, 1190 Vienna, Austria

${ }^{\text {\# } C u r r e n t ~ A d d r e s s: ~ I P B S, ~ C N R S, ~ U M R ~ 5089, ~} 205$ Route de Narbonne, 31077 Toulouse, France

*Corresponding author: BioCeV - Institute of Microbiology, Prumyslova 595, Vestec, 252 42, Czech Republic.E-mail: pman@biomed.cas.cz 


\begin{abstract}
Background: Cellobiose dehydrogenase $(\mathrm{CDH})$ is a fungal extracellular oxidoreductase which fuels lytic polysaccharide monooxygenase with electrons during cellulose degradation. Interdomain electron transfer between the flavin and cytochrome domain in $\mathrm{CDH}$, preceding the electron flow to lytic polysaccharide monooxygenase, is known to be $\mathrm{pH}$ dependent, but the exact mechanism of this regulation has not been experimentally proven so far.
\end{abstract}

Methods: To investigate the structural aspects underlying the domain interaction in $\mathrm{CDH}$, hydrogen/deuterium exchange (HDX-MS) with improved proteolytic setup (combination of nepethesin-1 with rhizopuspepsin), native mass spectrometry with ion mobility and electrostatics calculations were used.

Results: HDX-MS revealed $\mathrm{pH}-$ dependent changes in solvent accessibility and hydrogen bonding at the interdomain interface. Electrostatics calculations identified these differences to result from charge neutralization by protonation and together with ion mobility pointed at higher electrostatic repulsion between $\mathrm{CDH}$ domains at neutral $\mathrm{pH}$. In addition, we uncovered extensive O-glycosylation in the linker region and identified the long-unknown exact cleavage point in papain-mediated domain separation.

Conclusions: Transition of CDH between its inactive (open) and interdomain electron transfer-capable (closed) state is shown to be governed by changes in the protein surface electrostatics at the domain interface. Our study confirms that the interdomain electrostatic repulsion is the key factor modulating the functioning of $\mathrm{CDH}$.

General significance: The results presented in this paper provide experimental evidence for the role of charge repulsion in the interdomain electron transfer in cellobiose dehydrogenases, which is relevant for exploiting their biotechnological potential in biosensors and biofuel cells. 


\title{
Keywords
}

cellobiose dehydrogenase; direct electron transfer; electrostatic interaction; hydrogen/deuterium exchange; ion mobility; mass spectrometry

\author{
Abbreviations \\ $\mathrm{CDH}$, cellobiose dehydrogenase; $\mathrm{DH}$, dehydrogenase domain; CYT, cytochrome domain; \\ HDX-MS, hydrogen/deuterium exchange mass spectrometry; IET, interdomain electron \\ transfer; IM-MS, ion mobility mass spectrometry
}

\section{Introduction}

Cellobiose dehydrogenase (CDH, cellobiose:(acceptor) 1-oxidoreductase, E.C. 1.1.99.18) is the only known extracellular flavocytochrome. It is produced by wood-degrading and phytopathogenic fungi from the Basidiomycota and Ascomycota phyla. [1] $\mathrm{CDH}$ participates in the early events of lignocellulose degradation, as found in several fungi at the transcriptional and translational levels. $[2,3]$ The enzyme is also highly relevant from the biotechnological point of view as CDH forms a promising component for third generation biosensors and biofuel cells based on direct electron transfer. [4-6] Additionally, $\mathrm{CDH}$ is a crucial enzyme in the process of cellulose depolymerization to shorter saccharides [7,8], that are envisaged as a potential source of renewable energy replacing fossilbased fuels. [9]

From a structural point of view, $\mathrm{CDH}$ is a monomeric enzyme consisting of two domains joined by a flexible linker region. The larger dehydrogenase $(\mathrm{DH})$ domain carries FAD as a cofactor while the smaller cytochrome (CYT) domain contains a heme $b$. The catalytic cycle of cellobiose dehydrogenase is divided into a reductive and an oxidative half-reaction. During the reductive half-reaction, $\beta$-Dcellobiose is oxidized at the anomeric $\mathrm{C} 1$ carbon to yield $\delta$-lactone, which is further hydrolyzed in bulk water to the corresponding carboxylic acid. [10] Due to the higher redox potential of the heme $b$ an electron from the catalytic center (FAD) can be delivered to the CYT domain during the oxidative half-reaction. The electrons are finally channeled from the heme cofactor to a terminal electron acceptor, which can be either a small molecule or a protein partner such as lytic polysaccharide monooxygenase (LPMO). [11] For such an electron flow to proceed, the key reaction is the interdomain electron transfer (IET) between FAD and heme within the CDH molecule. This transfer occurs only under acidic solution conditions as has been observed since early studies. [12] While the specific $\mathrm{pH}$ optima for this reaction differ between $\mathrm{CDHs}$ from different organisms $[4,13,14]$, both FAD and heme are in general reduced rapidly in the presence of cellobiose at slightly acidic $\mathrm{pH}$. At neutral $\mathrm{pH}$ only FAD reduction is fast, while the IET and the heme reduction are extremely slow. $[12,15]$ Based on these findings a theory of $\mathrm{CDH}$ functioning was proposed, in which the close contact of the two domains is necessary for the electrons to be transferred directly between the cofactors. Physical separation of CDH domains on the other hand prevents the IET to occur and stops the electron flow to the interaction partner. [16]

Detailed knowledge of the structure and dynamics of $\mathrm{CDH}$ is a prerequisite for improving of its biotechnological potential and tailoring its enzymatic properties. Most likely due to its dynamic nature and high degree of modification, the only high resolution structures available for $\mathrm{CDH}$ were derived from isolated dehydrogenase [17] and cytochrome [18] domains from Phanerochaete chrysosporium. Until recently, these structures thus served as a modelling template for the full-length protein. However, paper describing high-resolution X-ray structures of two conformations of CDH was published in 2015. [19] This study characterized two different full-length CDH molecules (proteins originating from Myriococcum thermophilum - Mt CDH and Neurospora crassa - NcCDH). Crystals were obtained at $\mathrm{pH} \sim 6.5$, which is for $N c \mathrm{CDH}$ close to its $\mathrm{pH}$ optimum while for $M t \mathrm{CDH}$ this is an intermediate value between its active and inactive form. [14] Interestingly, the enzymes crystallized in different conformations, each representing one conformational state predicted to occur during $\mathrm{pH}$ mediated interdomain cross-talk in $\mathrm{CDH}$. The static pictures of $\mathrm{X}$-ray structures were further 
complemented by small angle X-ray scattering (SAXS) data, which allowed estimating the occurrence of different conformers in the system. This analysis showed that both forms (closed and open) of CDH (resembling the crystal forms of $\mathrm{Mt} \mathrm{CDH}$ and $\mathrm{NcCDH}$, respectively) are simultaneously present among other conformers. [19] Also, molecular modelling and domain docking approaches suggest that the molecular mechanism preventing the domain interaction and IET at neutral $\mathrm{pH}$ is the repulsion of negative charge patches at the domain-domain interface. [14] The role of charged residues around the interdomain interface was further highlighted by our recent studies where the effect of calcium ions on the IET at neutral $\mathrm{pH}$ was described. We showed that calcium enables IET even at higher $\mathrm{pH}$ values and this effect can be explained by shielding of the negative charge patches, mostly localized on the interface of DH and CYT domains. [14,20]

In order to structurally validate the theory of $\mathrm{pH}$-regulated domain charge repulsion and to better understand the mechanisms involved in controlling the IET, we focused our present study on determining what effects the $\mathrm{pH}$ has on the conformation and dynamics of $\mathrm{CDH}$ in solution. For this, we selected $\mathrm{CDH}$ from $M$. thermophilum, which was successfully crystallized in its closed (IETcapable) form. [19] As the study of proteins under different $\mathrm{pH}$ values can be quite challenging we approached this task using a combination of techniques based on structural mass spectrometry. Here we utilized hydrogen/deuterium exchange coupled with mass spectrometry (HDX-MS) and native ion mobility mass spectrometry (IM-MS). Although these techniques usually have lower structural resolution and do not directly provide 3D structures, they are well suited for monitoring conformational changes of molecules in their various functional states and are theoretically unlimited by the size and complexity of the studied systems. [21-23] In addition, HDX-MS can work under native-like conditions, with the studied protein directly in aqueous environment and at low concentrations, which rules out possible artifacts created by concentrated protein solutions. [24] If interpreted in the context of high-resolution structures, structural MS data add another dimension to our understanding of the studied biological system.

Here we provide experimental evidence for the "charge repulsion model" of the $\mathrm{pH}$-dependent interdomain electron transfer in $\mathrm{CDH}$. We show that at lower $\mathrm{pH}$ values, the areas on the interface of CYT and DH domains get protonated and change their organization with respect to hydrogen bonding as evidenced by increased deuteration kinetics. We also demonstrate that this property is an intrinsic behavior of each domain and does not depend on their physical contact.

\section{Materials and methods}

\subsection{Chemicals and enzymes}

All chemicals were obtained from Sigma-Aldrich unless stated otherwise. Endoglycosidase Endo Hf was supplied by New England Biolabs. Porcine pepsin (3.200-4.500 units/mg) was bought from Sigma-Aldrich, whereas nepenthesin-1 and rhizopuspepsin were recombinantly prepared and purified as described previously. [25,26] All the three proteases were immobilized in-house onto POROS20AL perfusion resin (Applied Biosystems) using a published procedure. [26-28]

\subsection{Protein production and deglycosylation}

Full-length cellobiose dehydrogenase from Myriococcum thermophilum (Uniprot ID: A9XK88, without the 21-aminoacid N-terminal signal sequence) was recombinantly expressed in Pichia pastoris and purified as described previously. [29] In order to prepare the two individual $M t \mathrm{CDH}$ domains, part of the full-length sample was treated by papain and separated using ion exchange chromatography. $[19,30]$ Enzymatic activity of the $M t \mathrm{CDH}$ preparations was tested using the standard electron transfer assays based on monitoring the reduction of 2,6-dichloroindophenol and cytochrome $c$ [31,32], while protein concentration was determined by Pierce BCA protein assay kit (Thermo Fisher).

\subsection{Non-denaturing deglycosylation}

For analyses requiring $\mathrm{N}$-deglycosylated protein, $M t \mathrm{CDH}$ was treated by glycosidase Endo $\mathrm{Hf}(\mathrm{New}$ England Biolabs) as described elsewhere.[20] Briefly, $M t \mathrm{CDH}$ was incubated overnight at $37^{\circ} \mathrm{C}$ in 50 $\mathrm{mM}$ sodium acetate buffer $\mathrm{pH} 5.4$ with $15 \mathrm{U}$ Endo Hf per $1 \mu \mathrm{g}$ of protein. 


\subsection{Post-translational modification characterization}

Analysis of the disulfide bond pattern was performed utilizing a method developed previously. [33] Proteases used for digestion of the protein were Trypsin Gold MS grade (Promega), chymotrypsin, Asp-N and Glu-C (all three sequencing grade from Roche) at $5 \mathrm{ng} / \mu 1$ concentration.

To characterize other post-translational modifications and identify papain cleavage site, native or Endo Hf treated samples were resolved on SDS electrophoresis. Proteins were further in-gel reduced by tris(2-carboxyethyl)phosphine (TCEP), alkylated with iodoacetamide and digested by specific proteases (Trypsin, Asp-N and Glu-C).

Samples were subjected to an LC-MS/MS analysis using collision-induced fragmentation on an Fourier transform ion cyclotron resonance (FT-ICR) mass spectrometer equipped with 15T superconducting magnet (SolariX XR, Bruker Daltonics). Tandem mass spectra were searched against a single-protein database containing the $M t \mathrm{CDH}$ sequence using Mascot algorithm with carbamidomethylation (Cys, +57.0215), N-acetylhexosamine (Asn, +203.0794), hexose (Ser, Thr, +162.0528 ) and pyroglutamic acid (N-terminal Gln, -17.0265) set as variable modifications. N- and Oglycosylation sites and glycan structures were identified by manual searching of the data and with the help of the GlycoPeptideSearch engine. [34]

Intact mass measurements of CYT domain were performed using sample desalted by benchtop gel filtration $(0.5 \mathrm{ml}$ Zeba Spin column with $7 \mathrm{kDa}$ cut-off, Thermo Fisher Scientific) and diluted to 10 $\mu \mathrm{M}$ into 50\% ACN / 0.1\% TFA. Protein was ionized by ESI and analyzed using 15T FT-ICR mass spectrometer operated in positive ion mode with continuous accumulation of selected ions $(1370 \pm 130$ $\mathrm{m} / \mathrm{z}$ window).

\subsection{Hydrogen/deuterium exchange}

$M t \mathrm{CDH}$, either fully glycosylated or N-deglycosylated under native conditions, was transferred into either $50 \mathrm{mM}$ 4-morpholineethanesulfonate (MES) pH 5.4 or $50 \mathrm{mM}$ 4-morpholinepropanesulfonate (MOPS) $\mathrm{pH}$ 7.4. After 30 min preincubation, the H/D exchange workflow proceeded as described before. [20] Briefly, after 10-fold dilution by $\mathrm{D}_{2} \mathrm{O}$-based MES or MOPS buffer (pD 5.4 and pD 7.4, respectively) the deuteration was left to proceed at $21^{\circ} \mathrm{C}$ for $0.33,1,3,10,30,60,180$ and 300 min. Protein concentration during the exchange was $5 \mu \mathrm{M}$. Following the labelling, $50 \mu \mathrm{l}$ aliquots were quenched by the addition of $50 \mu \mathrm{l} 6 \mathrm{M}$ guanidine, $0.9 \mathrm{M}$ TCEP and $1 \mathrm{M}$ glycine $\mathrm{pH} 2.4$, and incubated for $10 \mathrm{~min}$ at $0{ }^{\circ} \mathrm{C}$ before being rapidly frozen in liquid nitrogen as optimized previously for proper unfolding and digestion efficiency. [20]

Upon quick thawing, the quenched samples were injected into an LC system described in detail before [20] and consisting of immobilized protease column, peptide desalting microtrap (Michrom Bioresources) and analytical chromatographic column Jupiter C18 $(0.5 \times 5 \mathrm{~mm}, 5 \mu \mathrm{m}, 300 \AA$; Phenomenex). Proteases used for the digestion were either porcine pepsin, recombinant rhizopuspepsin [26] or recombinant nepenthesin-1 [28]. Digestion was carried out in $0.4 \%$ formic acid in water with constant flow of $100 \mu \mathrm{l} / \mathrm{min}$ for pepsin column and $200 \mu \mathrm{1} / \mathrm{min}$ when nepenthesin- 1 and rhizopuspepsin columns were used together, joined serially by a PEEK column coupler (IDEX Health\&Science). After digestion, peptides were desalted $(3 \mathrm{~min}$ ) before being eluted onto the analytical column operated at flow rate $15 \mu \mathrm{l} / \mathrm{min}$. Peptides were separated by a linear gradient of 10 $35 \% \mathrm{~B}$ in $12 \mathrm{~min}$, followed by a jump to $99 \% \mathrm{~B}$, where solvent $\mathrm{A}$ was $0.2 \%$ formic acid $/ 2 \%$ acetonitrile in water and $\mathrm{B}$ was $95 \%$ acetonitrile $/ 0.2 \%$ formic acid in water. All the fluidic pathway, protease and analytical columns were kept at $0^{\circ} \mathrm{C}$ to keep the deuterium back-exchange to a minimum. The LC system was directly interfaced to an electrospray ionization source of a 15T FT-ICR mass spectrometer. The instrument was operated either in LC-MS/MS mode (peptide identification) or LCMS mode (analysis of deuterated samples). Tandem mass spectrum data were searched against a MASCOT database containing the sequence of $M t \mathrm{CDH}$. HDX-MS data were processed using DeutEx, an in-house developed program. The statistical significance of the reported differences was evaluated 
similarly as described before.[35] Based on replicate measurements of deglycosylated full-length CDH at $\mathrm{pH} 5.4$ and 7.4, the average standard deviation of deuteration was determined to be less than $0.1 \mathrm{Da}$. Therefore the differences can be considered as significant, if they exceed this threshold three times. No correction for back-exchange was performed and thus the values are reported as relative.

\subsection{Native mass spectrometry and ion mobility}

Fully glycosylated $M t \mathrm{CDH}$ was transferred into $200 \mathrm{mM}$ ammonium acetate $\mathrm{pH} 5.4 \mathrm{or} \mathrm{pH} 7.4$ by two cycles of benchtop size exclusion chromatography $(0.5 \mathrm{ml}$ Zeba Spin column with $7 \mathrm{kDa}$ cut-off). Desalted sample was then introduced into a Synapt G2 HDMS mass spectrometer (Waters) through an automated nanoelectrospray microfluidic injection system (Triversa Nanomate, Advion). Voltage on the electrospray tip was kept at $1.75 \mathrm{kV}$, while the mass spectrometer parameters were tuned to favor transmission of intact $\mathrm{MtCDH}$ molecule with minimal ion activation in the gas phase. Specifically, the instrument acquired data in the $\mathrm{m} / \mathrm{z}$ range $1,000-10,000$, with backing pressure kept at 6 mbar while the extraction cone voltage, trap collision energy and trap bias voltage were set to $4 \mathrm{~V}, 10 \mathrm{~V}$ and $40 \mathrm{~V}$, respectively. For collision induced activation experiments, the sample cone voltage was systematically increased from $130 \mathrm{~V}$ to $180 \mathrm{~V}$ in $5 \mathrm{~V}$ increments to gradually activate the ions in a controlled manner. The ion mobility settings were optimized to provide good separation with the drift time of $M t \mathrm{CDH}$ ions kept in the second third of the accessible drift time range. Travelling wave height and wave velocity were set to $40 \mathrm{~V}$ and $910 \mathrm{~m} / \mathrm{s}$, while the gas flows in the helium and ion mobility cells were kept at 100 and $25 \mathrm{ml} / \mathrm{min}$, respectively.

\subsection{Calculation of protein surface charge}

For the simulation of protein surface electrostatics, structures of cytochrome and flavin domain were individually extracted from the crystallographic structure of full-length $M t C D H$ (PDB ID: 4QI6). [19] Hydrogen atoms were assigned to the structures at $\mathrm{pH} 5.4$ and $\mathrm{pH} 7.4$ by PROPKA algorithm [36] used by PDB2PQR web server [37,38] during the preparation of molecules for calculations. The simulations of molecular electrostatics were then performed using the Adaptive Poisson-Boltzmann Software package (APBS) [39] and the results were visualized in PyMOL 1.8.0 (Schrödinger) with APBS Tools 2.1 plugin.

\section{Results and discussion}

\subsection{MtCDH is extensively post-translationally modified}

Detailed knowledge of protein primary structure is a prerequisite for successful protein characterization using HDX-MS. Therefore, it was necessary to characterize post-translational modifications present in the $M t \mathrm{CDH}$. This proved especially important as $M t \mathrm{CDH}$ expressed in Pichia pastoris has been shown to differ from $M t \mathrm{CDH}$ produced by the fungus itself. Variations in the molecular mass are due to different $\mathrm{N}$-glycosylation pattern and possible O-glycosylation in the linker region, which was predicted by bioinformatic tools. [29]

Using LC-MS/MS analyses, we confirmed the primary amino acid sequence of $M t \mathrm{CDH}$ along with the $\mathrm{N}$-terminal processing occurring during protein maturation (first 21 amino acids in Uniprot ID: A9XK88) and the N-terminal pyroglutamylation. Furthermore, we determined the disulfide bond network for the full-length $\mathrm{MtCDH}$ (Fig. 1a). This proved to be in agreement with the recent crystal structure of $M t \mathrm{CDH}$ [19], but additionally confirmed the existence of the Cys167-Cys211 disulfide bridge, which is not directly observable in the X-ray density.

Concerning the glycosylation status, all the six potential N-glycosylation sites (Asn-119, 400, 437, 516, 671 and 678, present in Asn-Xxx-Ser/Thr consensus sequence) were shown to be modified with high-mannose type glycans (Fig. 1b). The only exception was the Asn671, which was only partially glycosylated. Moreover, we also confirmed that the predicted O-glycosylation is indeed present in the interdomain linker region. In the 42-amino acid long region (188-230) twelve potential O-glycosites 
(Ser and Thr residues) with up to twenty hexose units attached (Fig. 1c) were found. Based on the numbers of hexoses in overlapping peptides produced by trypsin, chymotrypsin, Asp-N and Glu-C proteases, it seems that a kind of clustered O-glycosylation sites is present in the $\mathrm{MtCDH}$ linker region. This situation resembles the hinge region in IgA antibodies, where a similar pattern of multiply glycosylated clustered serine and threonine residues was described. [40] The findings are corroborated by the crystal structure of $M t \mathrm{CDH}$, where some mannose units were found in the vicinity of the potential O-glycosylation sites (Ser195, Thr197, Thr204, Thr206 and Thr226). [19]

\subsection{Papain cleaves $M t C D H$ before the flavin domain}

It has long been known that it is possible to use papain to cleave the intact $\mathrm{MtCDH}$ molecule into its two constituent domains - CYT and DH. [30] However, the precise cleavage site remained unknown. After papain cleavage of full-length $M t \mathrm{CDH}$, the two domains were separated, further digested and analyzed as described in section 2.4.. Spectra of peptides generated by Asp-N and trypsin digests of the separated DH (Fig. 2a) and CYT domains (Fig.s 2b, 2c) show that the papain cleavage results at the separation of the two domains at either Val220 or Gly221. The presence of these two residues at the peptide termini in the specific protease digests proves that these are indeed the terminal peptides produced by the papain action. Otherwise, this would be in contradiction with the cleavage rules of the specific proteases used.

ESI-FT-ICR spectrum of the intact CYT domain corroborates these findings by showing peak distributions attributable to three types of mass differences (Fig. 2d). First, there is a mass difference of $162 \mathrm{Da}$, which arises from the heterogeneity in the number of hexose units in the O- and N-linked glycans present in the CYT domain. Second, the $57 \mathrm{Da}$ difference between peaks of the same glycosylation state signifies the presence (or absence) of the Gly221 residue at the C-terminus of the domain. Judging by the similar intensity of these two proteolytic products, we can conclude that the papain cleaves $\mathrm{N}$ - and C-terminally of this residue with roughly the same probability. Finally, peaks with the $18 \mathrm{Da}$ difference show partial hydrolysis of the N-terminal pyroglutamic acid. The high resolution of FT-ICR mass spectrometer also allowed us to determine the precise monoisotopic masses of intact CYT domain proteoforms. For the most abundant form in Fig. $2 d$ the determined mass 25459.387 Da (mass error $\pm 0.3 \mathrm{ppm}$ ) corresponds to $\mathrm{MtCDH}$ amino acids $1-220$ with $\mathrm{N}$-terminal glutamine converted to pyroglutamic acid, two $\mathrm{N}$-acetylhexosamines and eleven hexoses. It also confirms that in the CYT domain three are disulfide bonds formed.

\subsection{Increasing HDX-MS spatial resolution by alternative aspartic proteases}

Having characterized the modifications present on the $M t \mathrm{CDH}$ molecule, we focused on optimizing the HDX-MS method for $M t \mathrm{CDH}$ structural studies. In previous work, we described HDX-MS compatible conditions for the pepsin digestion of $M t \mathrm{CDH}$. [20] By incubating the deuterium labeled $\mathrm{N}$-deglycosylated $\mathrm{MtCDH}$ in a quenching buffer containing denaturing and reducing agents on ice prior to the online pepsinolysis, we achieved almost complete (93\%) coverage of the protein missing basically only the O-glycosylated linker region.

However, some of the peptides produced by this immobilized pepsin digestion of $M t \mathrm{CDH}$ were quite long, leading to less precise localization of the deuteration changes. (Fig. S1, blue) Therefore, we selected alternative aspartic proteases that are complementary in their cleavage preferences to the classical porcine pepsin, in order to increase the spatial resolution and other digestion parameters (peptide redundancy, sequence coverage, etc.). The two proteases, recombinant nepenthesin-1 [28] and rhizopuspepsin [26], were immobilized onto POROS-20AL resin and filled into individual columns. These were coupled serially (with nepenthesin preceding the rhizopuspepsin) in an online LC setup. Initially, we observed that the efficiency of the combined digestion was too high, leading to an overdigestion and a lower coverage of several protein regions due to the production of very short peptides. We limited the digestion time by doubling the sample flow rate through the protease columns to $200 \mu \mathrm{l} / \mathrm{min}$. The combined digestion resulted in a slight increase in total sequence coverage (95\%) 
but most importantly, it also led to an increase in the spatial resolution and other HDX-MS protease efficiency metrics (Table 1). This occurred by introducing new cleavage sites different from those obtained by pepsin, at both "pepsin-like" (Trp, Phe, Leu) as well as "non pepsin-like" cleavage sites (basic residues Lys, Arg, His). [26,28] The structural resolution increase is apparent when looking at the sequence coverage map in several parts of the $M t \mathrm{CDH}$ molecule - e.g. in regions 1-46, 439-478, 586-616 or 690-720 (Fig. S1). Taking a closer look at one of these regions - the ultimate N-terminus of the cytochrome domain - we can clearly see increase in spatial resolution (Fig. 3). Originally, with immobilized pepsin (Fig. 3a - blue bars) the changes in deuteration could only be mapped onto two rather long peptides (Fig. 3b). The shorter and more overlapping peptides produced by nepenthesin in combination with rhizopuspepsin (Fig. $3 \mathrm{a}$ - red bars) made it possible to narrow down the structurally perturbed regions to the two loops in this part of the domain (Fig. 3c).

From a practical perspective, it is important to mention that even though nepenthesin- 1 was shown to be very sensitive to reducing and denaturing agents in solution $[28,41,42]$, the immobilization and the flow-through setup of the digestion greatly stabilizes this enzyme [28]. Indeed, during the course of this study including hundreds of injection no adverse effect on the digestion efficiency was observed.

\subsection{HDX-MS study of pH-dependent domain interaction}

In order to study the structural dynamics of $M t \mathrm{CDH}$ in solution, we utilized the HDX-MS method as described in section 2.5. First of all, we checked whether the removal of N-linked glycans by the Endo Hf treatment had any influence on the structure of $M t \mathrm{CDH}$. Although the glycosylation might be important for the long-term as well as thermal stability of $M t \mathrm{CDH}$, HDX-MS data showed that the $\mathrm{N}$-deglycosylation performed under non-denaturing conditions causes only local structure changes slightly above the significance threshold. These were observed in the vicinity of the N-glycosylation sites where the protein became slightly more solvent accessible upon glycan removal. However, it still can be concluded that despite those local changes the deglycosylation had only negligible effect on the overall structure of $M t \mathrm{CDH}$. (Fig. S2) This was corroborated by spectrophotometric assays proving that the removal of $\mathrm{N}$-linked glycans did not change $M t \mathrm{CDH}$ 's enzymatic activity as determined by the cytochrome c assay and as observed in previous work performed with deglycosylated $M t \mathrm{CDH}$ immobilized on electrodes.[32,43]

Moreover, as the deglycosylation was performed by Endo Hf glycosidase rather than PNGase F, the proximal $\mathrm{N}$-acetylhexosamine of each glycan remained covalently attached to an asparagine residue. This was desirable as it prevented possible changes in the hydrogen bonding networks of the $M t \mathrm{CDH}$ molecule through the conversion of neutral Asn to acidic Asp residues by the action of PNGase F. As a result, we performed further HDX-MS analyses with the more homogeneous N-deglycosylated form. To gain insight into the mechanism of $M t \mathrm{CDH}$ regulation by $\mathrm{pH}$, we used HDX-MS to monitor the enzyme under two different $\mathrm{pH}$ values. Based on previous results, we selected $\mathrm{pH} 5.4$, which is close to the $\mathrm{pH}$ optimum of IET for class II cellobiose dehydrogenases, as an "active" state with the two domains presumably coming into close contact. In contrast, $\mathrm{pH} 7.4$, with negligible IET, was chosen to represent an "inactive" state. [14,44,45]

Since hydrogen/deuterium exchange strongly depends on solution $\mathrm{pH}$, the deuteration kinetics at the two selected $\mathrm{pH}$ values are not directly comparable. [46-49] Such a comparison is however possible after simple correction [22,50-52] which, in this particular case ( $\mathrm{pH} 5.4$ and 7.4), can be done by offsetting the deuteration curve for the higher $\mathrm{pH}$ by a factor of $100\left(10^{\Delta \mathrm{pD}}\right)$ on the logarithmic time scale.

The significant differences in deuteration between the $M t \mathrm{CDH}$ at the two $\mathrm{pH}$ values mapped on the surface of DH and CYT domains are shown in Fig. 4 (complete dataset is shown in Fig. S3). The structures were taken from the crystallographic model of the intact $M t \mathrm{CDH}$ molecule (PDB ID: 4QI6; Fig. 4 inset) and the domains were rotated, so the interdomain interface can be clearly seen. Regions 
with detected $\mathrm{pH}$-dependent changes in deuteration are color coded in different shades of blue, with the biggest differences represented in the darkest blue.

For both domains, most of the regions affected by the $\mathrm{pH}$-change are localized around their expected interaction interface with some changes on the negatively charged fungal carbohydrate binding module as well. On the cytochrome domain, these regions covered the majority of its DH-facing side with the most affected parts mapped to loops forming the active center around the heme cofactor (Fig. $4 \mathrm{~b})$. Similarly, for the flavin domain, the majority of $\mathrm{pH}$-perturbed regions was found around the edge of the cavity leading to the FAD cofactor in the enzymatic active site (Fig. 4a). These identified regions fit well into the context of the solved crystal structure of the whole $M t \mathrm{CDH}$ in its closed conformation, where the cytochrome domain fits into the cavity on the flavin domain enabling a propionate group of the heme to contact the buried flavin resulting in the transfer of electrons. [19]

Surprisingly, in all the protein regions influenced by the change of $\mathrm{pH}$, we observed increased deuteration at the lower $\mathrm{pH}$ at which the two domains interact (Fig. 4c, red and dark blue curves). This was unexpected, since in most cases the interaction of proteins or protein domains leads to a decrease in deuteration, resulting from the exclusion of solvent and formation of interprotein hydrogen bonds. However, it has been pointed out by other groups [53-55] that a protein/ligand interaction can result in a variety of observable outcomes in HDX-MS ranging from the canonical protection to deprotection due to conformational rearrangements. Interestingly, highly dynamic or amino acid side chain mediated interactions in rigid systems may also fail to provide any change in deuteration. In order to prove whether the observed changes are caused by the interdomain contact or are instead caused by the changes in $\mathrm{pH}$, we repeated the HDX-MS experiments with individual separated domains of $M t \mathrm{CDH}$. Since the IET capability of $\mathrm{CDH}$ is diminished if the two domains are separated, this model provides a good basis for such experiment as it uncouples the two phenomenons under consideration. The changes in deuteration around the interdomain interacting surface observed on the individual domains proved to be identical as those detected for the full-length protein (Fig. 4c). The only exception was slightly increased $\mathrm{pH}$ independent protection of some regions in the separated CYT domain. This is likely associated with the physical separation of DH and CYT domains, which leads to changes of the overall flexibility and/or accessibility of CYT domain (Fig. 4c, regions 2 and 3). Despite these changes in the CYT domain we can conclude that in the case of $\mathrm{Mt} \mathrm{CDH} \mathrm{pH}$-modulation, the observed protein backbone deprotection is in fact not directly caused by the domain contacting, but is instead a result of the $\mathrm{pH}$ change.

Of interest is also the lack of any detectable "classical" backbone amide protection, which is the most commonly observed deuteration scenario in HDX-MS, and normally leads to a deuteration decrease in the regions where two proteins interact. One reason for this unusual behavior may be the fact, that based on the crystal structure, the contact between the two domains seems to be mainly mediated by protruding heme propionate moieties on CYT domain and amino acid side chains on DH domain. [19] Also, the deuteration behavior suggests an inherent high flexibility and rapid dynamics of $M t \mathrm{CDH}$ in solution. It seems that the system does not go through a well-defined transition between its active (closed) and inactive (open) states, but rather occupies a plethora of semi-open transitional conformations. The probability of the system to move into its closed IET-competent conformation is then determined by the interdomain electrostatics, which is in turn governed by the $\mathrm{pH}$ of the solution. This explanation of the $M t \mathrm{CDH}$ dynamics are in accordance with the data obtained by small-angle Xray scattering measurements reported together with the crystal structure of full-length CDHs. [19] Here, the SAXS measurements revealed that the open and closed conformations represent only small subset of the populations present in the solution at $\mathrm{pH} 4.5$ with a range of other conformer clusters present concomitantly. [19] Therefore, as the IET-competent state seems to be only occupied for short periods of time and, as no more than 20-30\% of the molecules [19] seem to be present in the closed conformation even for the $\mathrm{Mt} \mathrm{CDH}$ active state, it is hardly surprising that only the deuteration changes induced by protonation are directly observable by HDX-MS. 
Furthermore, we correlated the HDX-MS data with protein surface electrostatic charge distributions modelled computationally for both $M t \mathrm{CDH}$ domains at $\mathrm{pH} 5.4$ and 7.4. In protein regions where the most significant deuteration changes were discovered by HDX-MS, we also observed neutralization of negative charge on protein surface due to protonation at the more acidic pH. (Fig. 5a and c) The neutralization is most prominent on the DH domain and especially in its part around and on the sides of the cavity leading to the FAD cofactor in the active site. Thus, the domain surface effectively repolarizes in this region from negative charge at $\mathrm{pH} 7.4$ to neutral or slightly positive values at $\mathrm{pH}$ 5.4, suppressing the electrostatic repulsion between the two domains. The involved protonation of amino acid side chains, which in turn leads to the rearrangement of hydrogen bonding networks in the affected regions of $M t \mathrm{CDH}$, is then offering the explanation for the unusual deuteration behavior of $M t \mathrm{CDH}$. As the hydrogen bonds are perturbed, the protein backbone amide hydrogens get more accessible for the exchange with deuterium from solution and/or the protein regions around the $\mathrm{Mt} \mathrm{CDH}$ interdomain interface slightly loosen structurally to better accommodate the domain-domain contact.

The magnitude of the observed surface electrostatic changes might seem surprising. Especially in the context of the intrinsic pKa constants for Asp and Glu residues which are 3.9 and 4.3, respectively. [56] Theoretically these residues should still be mostly deprotonated at $\mathrm{pH}$ 5.4. However, the above mentioned $\mathrm{pKa}$ values were determined for isolated freely accessible groups in short polyalanine peptides. In contrast, pKa values for Asp and Glu sidechains in real proteins are influenced significantly by their structural environment - namely by the level of amino acid burial, its involvement in hydrogen bonds or coulombic interactions with other charges. [56,57] Moreover, the APBS algorithm [57] used in this work provides the net electrostatic potential at individual places of the protein surface and thus sums various contributions from different ionizable groups, including not only Asp and Glu, but also His and other residues.

\subsection{Protein stability probed by IM-MS}

Finally, we attempted to validate the HDX-MS findings using native mass spectrometry coupled to ion mobility. This technique, when tuned carefully, can gently transfer macromolecules into the gas phase inside the mass spectrometer, while largely keeping the features of their tertiary and quaternary structure unperturbed. [58] Ion mobility then separates ions based on their size and shape, adding another dimension of information to molecular mass values obtained by MS.

The fully-glycosylated $M t \mathrm{CDH}$ was introduced into the mass spectrometer via a nanoelectrospray ionization interface from ammonium acetate solutions of $\mathrm{pH} 5.4$ and 7.4. Even though the presence of both $\mathrm{N}$ - and $\mathrm{O}$-glycosylation and their heterogeneity resulted in broad mass peaks, the drift time distributions extracted at both $\mathrm{pH}$ for the lowest charge state present $(16+$, mass window $6450 \pm 50$ Da) resulted in fairly narrow Gaussian curves (Fig. 6b). By comparing the drift time distributions for ions generated from solutions of $\mathrm{pH} 5.4$ and 7.4 (red and blue curves in Fig. 6b, respectively) no difference could be detected directly. This means that the ions produced from both $\mathrm{pH}$ conditions adopt conformation(s) of similar size in the gas phase. However, differences between the ions appeared when we started to increase their internal energy. This was achieved by gradually increasing the sample cone voltage in the ion source of the mass spectrometer, leading to faster acceleration and more collisions of the ions with residual gas molecules. As the accelerating voltage was incrementally increased from $130 \mathrm{~V}$ to $180 \mathrm{~V}$, the ions transitioned distinctly from the original conformation to a more compact one, marked $\mathrm{C} 1$ and $\mathrm{C} 2$ respectively (Fig. 6b). Most importantly, this transition proceeded differently depending on the $\mathrm{pH}$ of the solution $M t \mathrm{CDH}$ was electrosprayed from. The intermediate accelerating voltages clearly showed that the $M t \mathrm{CDH}$ ions formed from the more acidic solution collapsed more easily than those from solution of $\mathrm{pH}$ 7.4. This can be seen even more clearly in Fig. 6a, where a ratio of conformers "R" was plotted against the accelerating sample cone voltage 
increased at $5 \mathrm{~V}$ increments. The curves were constructed by fitting the drift time distributions at individual voltages with two Gaussian curves, leading to the ratio $\mathrm{R}$ of the area under the curve for the collapsed conformer $(\mathrm{C} 2)$ divided by the sum of areas under the curves of both conformers $(\mathrm{C} 2+\mathrm{C} 1)$. The resulting sigmoidal curves clearly show that $\mathrm{MtCDH}$ molecules ionized from $\mathrm{pH} 5.4$ solution require less activation energy to collapse. It should be also noted that exactly the same experiments were performed with the $\mathrm{N}$-deglycosylated form of $M t \mathrm{CDH}$ and provided identical result. Thus we can conclude that this behavior is not influenced by the N-glycosylation.

The observed collapse of $\mathrm{Mt} \mathrm{CDH}$ molecules upon gas phase activation is an interesting phenomenon, as most proteins tend to unfold and adopt more extended conformations during such collision-induced unfolding analyses as described above. [59,60] However, for some proteins with internal cavities, ionic collapse was observed preceding their unfolding/fragmentation. [60] Therefore, this could suggest that under both $\mathrm{pH}$ the $M t \mathrm{CDH}$ molecules are transferred into the gas phase in (at least partially) open conformation and as their internal energy increases, the molecules are forced into their closed state where the cytochrome domain protrudes into the cavity of the flavin domain. As the distribution of negative charges at the domain interface (especially on the DH domain) changes between the two $\mathrm{pH}$ states (see Fig. 5), the energy barrier of domain contacting stemming from the repulsion of patches of negative charge changes as well. The observed lower energy needed for the transition of ions at $\mathrm{pH} 5.4$ then means that the energy barrier is smaller for the closing of the domains at slightly acidic $\mathrm{pH}$, which agrees with both the surface electrostatics calculations and the $M t \mathrm{CDH}$ enzymatic $\mathrm{pH}$ profile data. It is important to point out here that at both $\mathrm{pH}$ values ions of identical charge state were compared. Therefore, the difference in ion stability could indeed be explained by a different repartitioning/localization of the same number of protons.

It is also necessary to mention at this point, that the strength of electrostatic interactions determined in the gas phase cannot be directly compared with the strength of forces acting in solution [61] as bulk water, that surrounds molecules in solution, attenuates the effects of electrostatic interactions by partially shielding the charges. The desolvation also leads to the complete absence of hydrophobic interactions in the gas phase. However, despite the fact that the strength of electrostatic interactions between the two domains may be slightly overestimated in vacuo, the difference in the electrostatic stability of $\mathrm{MtCDH}$ was clearly dependent on its protonation arrangement state.

Most importantly, together with HDX-MS experiments and computational electrostatics calculations, which studied the system directly in solution or took the presence of solvent into account through calculation with implicit solvation [39], the experimental data show that the electrostatic repulsion is indeed the key factor in $\mathrm{pH}$-dependent regulation of the transient interaction of $M t \mathrm{CDH}$ domains in solution. This finding is also consistent with and experimentally supports the theory of domain charge repulsion proposed in previous studies, which described how the divalent cations bridge the patches of negative charge and enable the $\mathrm{Mt} \mathrm{CDH}$ domains to come into close contact necessary for IET, even at neutral $\mathrm{pH}$. [14,20]

\section{Conclusions}

In this work mass spectrometry was used to study $M t \mathrm{CDH}$ in solution. Primary structure analysis confirmed extensive O-glycosylation in the flexible linker region and also answered the long-standing question of the exact site of papain cleavage, which has long been used to produce separate $M t \mathrm{CDH}$ domains. This enzymatic action was found to take place at both $\mathrm{N}$ - and C-terminal sides of Gly221 in the linker region.

HDX-MS was used to probe the conformational dynamics and the pH-regulated electron transfer function of $M t \mathrm{CDH}$. Here we benefited from improved proteolytic setup combining immobilized nepenthesin-1 [28] with rhizopuspepsin [26], which increased the digestion efficiency and spatial resolution. Together with computational electrostatics simulations HDX-MS provided a direct experimental evidence that the contacting of $M t \mathrm{CDH}$ domains in solution is regulated by the repulsion of patches of negative charge at the interdomain interface. This supports the charge repulsion theory of 
$\mathrm{CDH}$ functioning as proposed in previous works and in our study of the effects of divalent cations on the activity of $\mathrm{MtCDH}$ at neutral to alkaline $\mathrm{pH}$. [14,17,20] According to our current results, at acidic $\mathrm{pH}$, regions of negative charge mainly on the $\mathrm{DH}$ domain are neutralized by protonation, allowing the interaction with the CYT domain and enabling efficient interdomain electron transfer. This observation was also linked with results provided by native IM-MS, which corroborated the conclusion that electrostatic repulsion between domains is significantly stronger at higher $\mathrm{pH}$.

Also, our results indicate that the interdomain interaction in $\mathrm{MtCDH}$ is transient with many protein conformations existing concomitantly in the solution, as suggested by previous SAXS measurements. [19] This manifested itself in HDX-MS by the absence of "classical" protection by interaction. In the case of $\mathrm{Mt} \mathrm{CDH}$, only deprotection resulting from the protonation of amino acid sidechains was observed. Therefore, this enzymatic system reiterated the necessity of looking beyond the most common scenarios and outcomes when interpreting HDX-MS results. [53,54]

\section{Acknowledgements}

This project was supported by the Czech Science Foundation (Grant P206/12/0503) and the Austrian Science Foundation (Grant P12069). EU / MEYS funding sources (CZ.1.05/1.1.00/02.0109 and LQ1604) are acknowledged for setup and access to MS instrumentation. S.C. was supported by the CNRS and the University of Strasbourg and also thanks the GIS IBiSA, the Région Alsace and the Communauté Urbaine de Strasbourg for the financial support in purchasing the Synapt G2 HDMS system. J.S. acknowledges the Institut de Recherches Servier for supporting his doctoral fellowship.

\section{Appendix A. Supplementary data}

Supplementary data to this article can be found online at XXXX

\section{References}

[1] M. Zamocky, R. Ludwig, C. Peterbauer, B.M. Hallberg, C. Divne, P. Nicholls, D. Haltrich, Cellobiose dehydrogenase--a flavocytochrome from wood-degrading, phytopathogenic and saprotropic fungi, Curr. Protein Pept. Sci., 7 (2006) 255-280.

[2] C.M. Phillips, A.T. Iavarone, M.A. Marletta, Quantitative proteomic approach for cellulose degradation by Neurospora crassa, J. Proteome Res., 10 (2011) 4177-4185.

[3] J. Sun, C. Tian, S. Diamond, N. Louise Glassa, Deciphering transcriptional regulatory mechanisms associated with hemicellulose degradation in Neurospora crassa, Eukaryot. Cell, 11 (2012) 482-493.

[4] R. Ludwig, W. Harreither, F. Tasca, L. Gorton, Cellobiose dehydrogenase: A versatile catalyst for electrochemical applications, ChemPhysChem, 11 (2010) 2674-2697.

[5] R. Ludwig, R. Ortiz, C. Schulz, W. Harreither, C. Sygmund, L. Gorton, Cellobiose dehydrogenase modified electrodes: advances by materials science and biochemical engineering, Anal. Bioanal. Chem., (2013).

[6] A.K.G. Felice, C. Sygmund, W. Harreither, R. Kittl, L. Gorton, R. Ludwig, Substrate Specificity and Interferences of a Direct-Electron-Transfer-Based Glucose Biosensor, J. Diabetes Sci. Technol., 7 (2013) 669-677.

[7] C.M. Phillips, W.T. Beeson, J.H. Cate, M. a Marletta, Cellobiose dehydrogenase and a copperdependent polysaccharide monooxygenase potentiate cellulose degradation by Neurospora crassa, ACS Chem. Biol., 6 (2011) 1399-406.

[8] R.J. Quinlan, M.D. Sweeney, L. Lo Leggio, H. Otten, J.-C.N. Poulsen, K.S. Johansen, K.B.R.M. Krogh, C.I. Jørgensen, M. Tovborg, A. Anthonsen, T. Tryfona, C.P. Walter, P. Dupree, F. Xu, G.J. Davies, P.H. Walton, Insights into the oxidative degradation of cellulose 
by a copper metalloenzyme that exploits biomass components, Proc. Natl. Acad. Sci. U. S. A., 108 (2011) 15079-84.

[9] Z. Fan, W. Wu, A. Hildebrand, T. Kasuga, R. Zhang, X. Xiong, A novel biochemical route for fuels and chemicals production from cellulosic biomass, PLoS One, 7 (2012) 1-8.

[10] W.C. Higham, D. Gordon-Smith, E. Dempsey, P.M. Wood, Direct 'H NMR evidence for conversion of b-D-cellobiose to cellobionolactone by cellobiose dehydrogenase from Phanerochaete chrysosporium, Fed. Eur. Biochem. Soc., 351 (1994) 128-132.

[11] D. Kracher, S. Scheiblbrandner, A.K.G. Felice, E. Breslmayr, M. Preims, K. Ludwicka, D. Haltrich, V.G.H. Eijsink, R. Ludwig, Extracellular electron transfer systems fuel cellulose oxidative degradation, Science (80-. )., 3165 (2016) 1-13.

[12] M. Samejima, K.E.L. Eriksson, A comparison of the catalytic properties of cellobiose:quinone oxidoreductase and cellobiose oxidase from Phanerochaete chrysosporium, Eur. J. Biochem., 207 (1992) 103-107.

[13] W. Harreither, C. Sygmund, M. Augustin, M. Narciso, M.L. Rabinovich, L. Gorton, D. Haltrich, R. Ludwig, Catalytic properties and classification of cellobiose dehydrogenases from ascomycetes, Appl. Environ. Microbiol., 77 (2011) 1804-1815.

[14] D. Kracher, K. Zahma, C. Schulz, C. Sygmund, L. Gorton, R. Ludwig, Inter-domain electron transfer in cellobiose dehydrogenase: modulation by $\mathrm{pH}$ and divalent cations, FEBS J., 282 (2015) 3136-3148.

[15] K. Igarashi, I. Momohara, T. Nishino, M. Samejima, Kinetics of inter-domain electron transfer in flavocytochrome cellobiose dehydrogenase from the white-rot fungus Phanerochaete chrysosporium, Biochem. J., 365 (2002) 521-6.

[16] K. Igarashi, M. Yoshida, H. Matsumura, N. Nakamura, H. Ohno, M. Samejima, T. Nishino, Electron transfer chain reaction of the extracellular flavocytochrome cellobiose dehydrogenase from the basidiomycete Phanerochaete chrysosporium, FEBS J., 272 (2005) 2869-2877.

[17] B.M. Hallberg, G. Henriksson, G. Pettersson, C. Divne, Crystal structure of the flavoprotein domain of the extracellular flavocytochrome cellobiose dehydrogenase, J. Mol. Biol., 315 (2002) $421-434$.

[18] B.M. Hallberg, T. Bergfors, K. Bäckbro, G. Pettersson, G. Henriksson, C. Divne, A new scaffold for binding haem in the cytochrome domain of the extracellular flavocytochrome cellobiose dehydrogenase, Structure, 8 (2000) 79-88.

[19] T.-C. Tan, D. Kracher, R. Gandini, C. Sygmund, R. Kittl, D. Haltrich, B.M. Hällberg, R. Ludwig, C. Divne, Structural basis for cellobiose dehydrogenase action during oxidative cellulose degradation, Nat. Commun., 6 (2015) 7542.

[20] A. Kadek, D. Kavan, A.K.G. Felice, R. Ludwig, P. Halada, P. Man, Structural insight into the calcium ion modulated interdomain electron transfer in cellobiose dehydrogenase, FEBS Lett., 589 (2015) 1194-1199.

[21] J.R. Engen, Analysis of protein conformation and dynamics by hydrogen/deuterium exchange MS, Anal. Chem., 81 (2009) 7870-7875.

[22] P. Man, C. Montagner, H. Vitrac, D. Kavan, S. Pichard, D. Gillet, E. Forest, V. Forge, Accessibility changes within diphtheria toxin $\mathrm{T}$ domain upon membrane penetration probed by hydrogen exchange and mass spectrometry, J. Mol. Biol., 414 (2011) 123-134.

[23] E. Trabjerg, R.U. Jakobsen, S. Mysling, S. Christensen, T.J.D. Jørgensen, K.D. Rand, 
Conformational Analysis of Large and Highly Disulfide-Stabilized Proteins by Integrating Online Electrochemical Reduction into an Optimized H/D Exchange Mass Spectrometry Workflow, Anal. Chem., 87 (2015) 8880-8888.

[24] D. Rozbesky, P. Man, D. Kavan, J. Chmelik, J. Cerny, K. Bezouska, P. Novak, Chemical cross-linking and H/D exchange for fast refinement of protein crystal structure, Anal. Chem., 84 (2012) 867-70.

[25] A. Kadek, V. Tretyachenko, H. Mrazek, L. Ivanova, P. Halada, M. Rey, D.C. Schriemer, P. Man, Expression and characterization of plant aspartic protease nepenthesin-1 from Nepenthes gracilis, Protein Expr. Purif., 95 (2014) 121-128.

[26] M. Rey, P. Man, G. Brandolin, E. Forest, L. Pelosi, Recombinant immobilized rhizopuspepsin as a new tool for protein digestion in hydrogen/deuterium exchange mass spectrometry, Rapid Commun. Mass Spectrom., 23 (2009) 3431-3438.

[27] L. Wang, H. Pan, D.L. Smith, Hydrogen exchange-mass spectrometry: optimization of digestion conditions, Mol. Cell. Proteomics, 1 (2002) 132-138.

[28] A. Kadek, H. Mrazek, P. Halada, M. Rey, D.C. Schriemer, P. Man, Aspartic protease nepenthesin-1 as a tool for digestion in hydrogen/deuterium exchange mass spectrometry, Anal. Chem., 86 (2014) 4287-94.

[29] M. Zamocky, C. Schumann, C. Sygmund, J. O'Callaghan, A.D. Dobson, R. Ludwig, D. Haltrich, C.K. Peterbauer, Cloning, sequence analysis and heterologous expression in Pichia pastoris of a gene encoding a thermostable cellobiose dehydrogenase from Myriococcum thermophilum, Protein Expr Purif, 59 (2008) 258-265.

[30] G. Henriksson, G. Pettersson, G. Johansson, A. Ruiz, E. Uzcategui, Cellobiose oxidase from Phanerochaete chrysosporium can be cleaved by papain into two domains, Eur. J. Biochem., 196 (1991) 101-106.

[31] G. Canevascini, P. Borer, J.L. Dreyer, Cellobiose dehydrogenases of Sporotrichum (Chrysosporium) thermophile, Eur. J. Biochem., 198 (1991) 43-52.

[32] U. Baminger, S.S. Subramaniam, V. Renganathan, D. Haltrich, Purification and characterization of cellobiose dehydrogenase from the plant pathogen Sclerotium (Athelia) rolfsii, Appl. Environ. Microbiol., 67 (2001) 1766-74.

[33] P. Pompach, P. Man, D. Kavan, K. Hofbauerova, V. Kumar, K. Bezouska, V. Havlícek, P. Novák, Modified electrophoretic and digestion conditions allow a simplified mass spectrometric evaluation of disulfide bonds, J. Mass Spectrom., 44 (2009) 1571-8.

[34] P. Pompach, K.B. Chandler, R. Lan, N. Edwards, R. Goldman, Semi-automated identification of N-Glycopeptides by hydrophilic interaction chromatography, nano-reverse-phase LCMS/MS, and glycan database search, J. Proteome Res., 11 (2012) 1728-40.

[35] U. Leurs, B. Lohse, S. Ming, P.A. Cole, R.P. Clausen, J.L. Kristensen, K.D. Rand, Dissecting the binding mode of low affinity phage display peptide ligands to protein targets by hydrogen/deuterium exchange coupled to mass spectrometry, Anal. Chem., 86 (2014) 1173411741.

[36] M.H.M. Olsson, C.R. Søndergaard, M. Rostkowski, J.H. Jensen, PROPKA3: Consistent treatment of internal and surface residues in empirical pKa predictions, J. Chem. Theory Comput., 7 (2011) 525-537.

[37] T.J. Dolinsky, J.E. Nielsen, J.A. McCammon, N.A. Baker, PDB2PQR: an automated pipeline for the setup of Poisson-Boltzmann electrostatics calculations, Nucleic Acids Res., 32 (2004) 
W665-667.

[38] T.J. Dolinsky, P. Czodrowski, H. Li, J.E. Nielsen, J.H. Jensen, G. Klebe, N.A. Baker, PDB2PQR: expanding and upgrading automated preparation of biomolecular structures for molecular simulations, Nucleic Acids Res., 35 (2007) W522-525.

[39] N.A. Baker, D. Sept, S. Joseph, M.J. Holst, J.A. McCammon, Electrostatics of nanosystems: application to microtubules and the ribosome, Proc. Natl. Acad. Sci. U. S. A., 98 (2001) 10037-10041.

[40] K. Takahashi, S.B. Wall, H. Suzuki, A.D. Smith, S. Hall, K. Poulsen, M. Kilian, J.A. Mobley, B.A. Julian, J. Mestecky, J. Novak, M.B. Renfrow, Clustered O-Glycans of IgA1: Defining Macro- and Microheterogeneity by Use of Electron Capture/Transfer Dissociation, Mol. Cell. Proteomics, 9 (2010) 2545-2557.

[41] K. Kubota, Y. Metoki, S.B.P. Athauda, C. Shibata, K. Takahashi, Stability profiles of nepenthesin in urea and guanidine hydrochloride: comparison with porcine pepsin A, Biosci Biotechnol Biochem, 74 (2010) 2323-2326.

[42] M. Yang, M. Hoeppner, M. Rey, A. Kadek, P. Man, D.C. Schriemer, Recombinant Nepenthesin II for Hydrogen/Deuterium Exchange Mass Spectrometry, Anal. Chem., 87 (2015) 6681-7.

[43] R. Ortiz, H. Matsumura, F. Tasca, K. Zahma, M. Samejima, K. Igarashi, R. Ludwig, L. Gorton, Effect of deglycosylation of cellobiose dehydrogenases on the enhancement of direct electron transfer with electrodes, Anal. Chem., 84 (2012) 10315-10323.

[44] W. Harreither, P. Nicholls, C. Sygmund, L. Gorton, R. Ludwig, Investigation of the pHdependent electron transfer mechanism of ascomycetous class II cellobiose dehydrogenases on electrodes, Langmuir, 28 (2012) 6714-6723.

[45] W. Harreither, V. Coman, R. Ludwig, D. Haltrich, L. Gorton, Investigation of graphite electrodes modified with cellobiose dehydrogenase from the ascomycete Myriococcum thermophilum, Electroanalysis, 19 (2007) 172-180.

[46] A. Hvidt, A Discussion of the $\mathrm{pH}$ Dependence of the Hydrogen-Deuterium Exchange of Proteins, C. R. Trav. Lab. Carlsberg, 34 (1964) 299-317.

[47] Y. Bai, J.S. Milne, L. Mayne, S.W. Englander, Primary structure effects on peptide group hydrogen exchange, Proteins, 17 (1993) 75-86.

[48] Z. Zhang, D.L. Smith, Determination of amide hydrogen exchange by mass spectrometry: a new tool for protein structure elucidation, Protein Sci., 2 (1993) 522-31.

[49] P. Man, C. Montagner, G. Vernier, B. Dublet, A. Chenal, E. Forest, V. Forge, Defining the Interacting Regions between Apomyoglobin and Lipid Membrane by Hydrogen/Deuterium Exchange Coupled to Mass Spectrometry, J. Mol. Biol., 368 (2007) 464-472.

[50] S.J. Coales, S.Y. E, J.E. Lee, A. Ma, J.A. Morrow, Y. Hamuro, Expansion of time window for mass spectrometric measurement of amide hydrogen/deuterium exchange reactions, Rapid Commun. Mass Spectrom., 24 (2010) 3585-3592.

[51] J. Li, M. V Rodnin, A.S. Ladokhin, M.L. Gross, Hydrogen-Deuterium Exchange and Mass Spectrometry Reveal the $\mathrm{pH}$-Dependent Conformational Changes of Diphtheria Toxin $\mathrm{T}$ Domain, Biochemistry, 53 (2014) 6849-6856.

[52] B.T. Walters, P.F. Jensen, V. Larraillet, K. Lin, T. Patapoff, T. Schlothauer, K.D. Rand, J. Zhang, Conformational destabilization of immunoglobulin $\mathrm{G}$ increases the low $\mathrm{pH}$ binding 
affinity with the neonatal Fc receptor, J. Biol. Chem., 291 (2016) 1817-1825.

[53] M.A. Sowole, L. Konermann, Effects of protein-ligand interactions on hydrogen/deuterium exchange kinetics: canonical and noncanonical scenarios, Anal. Chem., 86 (2014) 6715-22.

[54] L. Konermann, A. Rodriguez, M.A. Sowole, Type 1 and type 2 scenarios in hydrogen exchange mass spectrometry studies on protein-ligand complexes, Analyst, 139 (2014) 60786087.

[55] J.R. Engen, Analysis of protein complexes with hydrogen exchange and mass spectrometry, Analyst, 128 (2003) 623-8.

[56] C.N. Pace, G.R. Grimsley, J.M. Scholtz, Protein ionizable groups: pK values and their contribution to protein stability and solubility, J. Biol. Chem., 284 (2009) 13285-13289.

[57] H. Li, A.D. Robertson, J.H. Jensen, Very fast empirical prediction and rationalization of protein pKa values, Proteins, 61 (2005) 704-21.

[58] B.T. Ruotolo, C. V Robinson, Aspects of native proteins are retained in vacuum, Curr. Opin. Chem. Biol., 10 (2006) 402-8.

[59] J.T.S. Hopper, N.J. Oldham, Collision Induced Unfolding of Protein Ions in the Gas Phase Studied by Ion Mobility-Mass Spectrometry: The Effect of Ligand Binding on Conformational Stability, J. Am. Soc. Mass Spectrom., 20 (2009) 1851-1858.

[60] Z. Hall, A. Politis, M.F. Bush, L.J. Smith, C. V Robinson, Charge-state dependent compaction and dissociation of protein complexes: insights from ion mobility and molecular dynamics, J. Am. Chem. Soc., 134 (2012) 3429-3438.

[61] S. Yin, Y. Xie, J.A. Loo, Mass Spectrometry of Protein-Ligand Complexes: Enhanced GasPhase Stability of Ribonuclease-Nucleotide Complexes, J. Am. Soc. Mass Spectrom., 19 (2008) 1199-1208. 


\section{Figure legends}

Fig. 1. Post-translational modifications of $M t \mathrm{CDH}$. (a) MS-derived pattern of disulfide bond linkages in the full-length protein. (b) All six N-glycosylation sites have a broad distribution of high-mannosetype glycans similar as the Asn119 site shown here. Blue square - N-acetylhexosamine, green circle hexose. (c) Overlapping Asp-N/trypsin-generated peptides show clustered O-glycosylation in the linker region of $M t \mathrm{CDH}$. The spectrum illustrates the O-glycan heterogeneity for the 206-247 protein region.

Fig. 2. Papain separates $M t \mathrm{CDH}$ domains by cleaving in the linker region. Asp-N/trypsin-generated peptides of DH (a) and CYT (b, c) domains show the cleavage to occur both N- and C-terminally to Gly221. (d) High-resolution ESI-FT-ICR spectrum of CYT domain shows a range of proteoforms differing by glycine residue $(\Delta 57 \mathrm{Da})$, number of hexoses $(\Delta 162 \mathrm{Da})$ and partial hydrolysis of $\mathrm{N}$ terminal pyroglutamic acid $(\Delta 18 \mathrm{Da})$. Inset shows baseline isotopic separation of the $1427.5890(18+)$ peak.

Fig. 3. Alternative aspartic protease cleavage increases the spatial resolution of HDX-MS. (a) Peptide mapping of the ultimate $\mathrm{N}$-terminus of $\mathrm{MtCDH}$. Individual peptides are drawn as blue bars (immobilized pepsin) and red bars (combined digestion by immobilized nepenthesin-1 and rhizopuspepsin). The additional cleavage sites enabled more precise localization of deuteration changes at the N-terminus of CYT domain (red and orange regions) with the combined irNep-1+irRpn digestion (c) compared to porcine pepsin (b).

Fig. 4. HDX-MS detected changes at the $M t \mathrm{CDH}$ interdomain interface between $\mathrm{pH} 5.4$ (active) and 7.4 (inactive). (a) DH domain with the most intense changes around the cusp of FAD cavity. Some perturbance was also seen for the fungal carbohydrate binding module (shown in teal color). (b) CYT domain with strongest $\mathrm{pH}$ effects detected around the heme cofactor at the DH-binding surface. For the sake of clarity the domains were rotated by $90^{\circ}$ in opposite directions from their natural orientation (inset). $M t \mathrm{CDH}$ structure (PDB ID: 4QI6) was colored according to the detected changes in deuteration. Grey means no difference while different shades of blue mark the deprotected regions with dark blue showing the biggest difference. Red regions show the connecting points of interdomain linker. (c) HDX-MS curves showing the number of exchanged deuterons for the individual regions of full-length $M t \mathrm{CDH}$ at $\mathrm{pH} 5.4$ and 7.4 (red and dark blue curves, respectively). Separated domains (black, dark green, magenta and orange) and their mixture (light green and cyan) behave similarly as full-length protein. $M t \mathrm{CDH}$ regions unaffected by $\mathrm{pH}$ show no difference $(5,7)$, while in the perturbed regions there is significant deprotection at the lower $\mathrm{pH}$.

Fig. 5. Protein surface electrostatics calculated for $M t \mathrm{CDH}$ domains - DH (top) and CYT (bottom) at $\mathrm{pH} 5.4(\mathrm{a}, \mathrm{c})$ and $\mathrm{pH} 7.4(\mathrm{~b}, \mathrm{~d})$. Electrostatic potential is shown as color gradient from red to blue (12 to $+12 \mathrm{kT} / \mathrm{e})$. Green lines mark areas of negative charge neutralization, which correlate with areas of the most pronounced differences detected by HDX-MS.

Fig. 6. Native IM-MS shows different electrostatic stability of $M t \mathrm{CDH}$ ionized from $\mathrm{pH} 5.4$ (red) and $\mathrm{pH} 7.4$ (blue). (a) Ions of fully-glycosylated $\mathrm{MtCDH}$ generated from lower $\mathrm{pH}$ collapse more easily with increasing sample cone voltage (SCV) than ions of the same charge state from higher $\mathrm{pH}$. Conformer ratio $\mathrm{R}=\mathrm{C} 2 /(\mathrm{C} 1+\mathrm{C} 2)$, where $\mathrm{C} 1$ and $\mathrm{C} 2$ are areas-under-curve in drift time distributions for individual activation voltages (b). Triplicate measurements were performed and the data are shown with their standard deviations.

\section{Table legends}


Table 1. MtCDH digestion metrics - Comparison of protein digestion by immobilized pepsin and the combination of nepenthesin-1 with rhizopuspepsin. iPep - immobilized porcine pepsin at $100 \mu \mathrm{l} / \mathrm{min}$; irNep-1 + irRpn - sequentially coupled immobilized recombinant nepenthesin-1 and recombinant rhizopuspepsin columns at $200 \mu \mathrm{l} / \mathrm{min}$; Cleavage efficiency - percentage of cleaved peptide bonds; redundancy score - average times a residue is covered by unique peptides. AAs - amino acids.

Table 1

\begin{tabular}{lcc}
\hline & iPep & irNep-1+ irRpn \\
\hline Number of peptides & 229 & 370 \\
Average peptide length (AAs) & 25.5 & 20.5 \\
Sequence coverage (\%) & 92.7 & 94.7 \\
Cleavage efficiency (\%) & 14.1 & 23.3 \\
Redundancy score & 7.23 & 9.41 \\
\hline
\end{tabular}




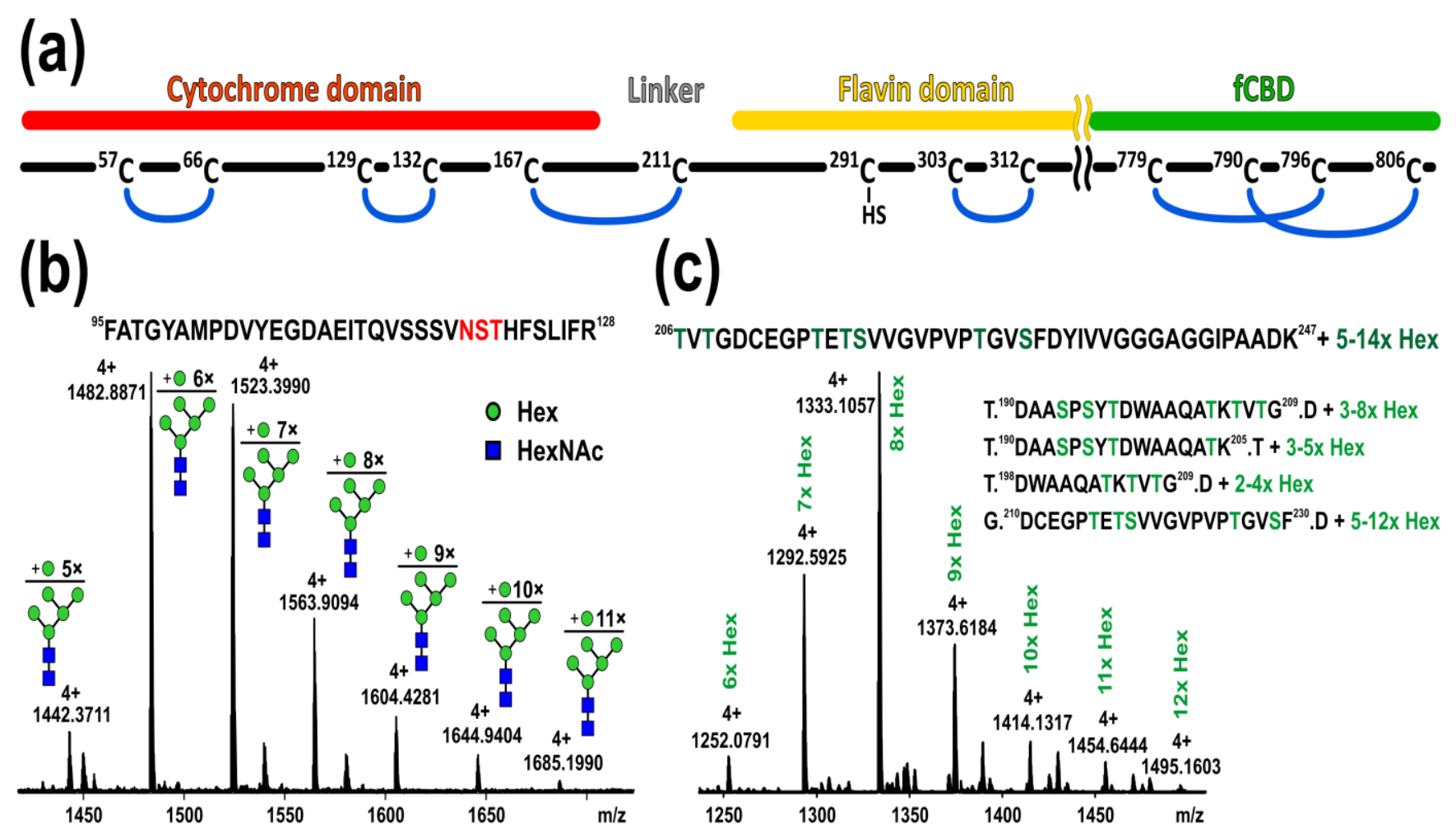

Figure 1 


\section{(a)}

$G^{222}$ VPVPTGVSF ${ }^{230} . D+2$ Hex

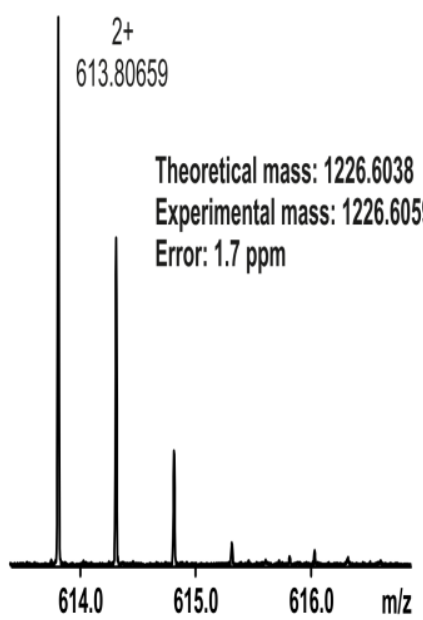

(b)

$K_{1}^{206}$ TVTGDCEGPTETSWVG ${ }^{21}, V+4$ Hex $K_{1}^{206}{ }^{26} V T G D C E G P T E T S W V^{20}, G+4$ Hex

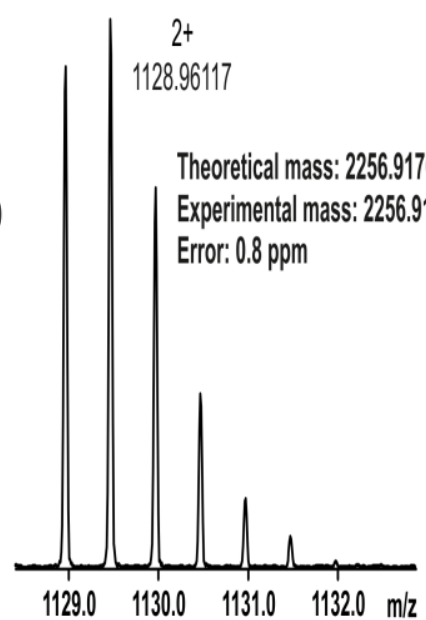

(C)

(d) ${ }_{\text {Gly }}^{457}$

$\leftrightarrow \Delta 162$

Hex

$\mid \begin{gathered}2+ \\ 1100.45151\end{gathered}$

Theoretical mass: 2199.8956

Experimental mass: 2199.8957

Error: 0 ppm

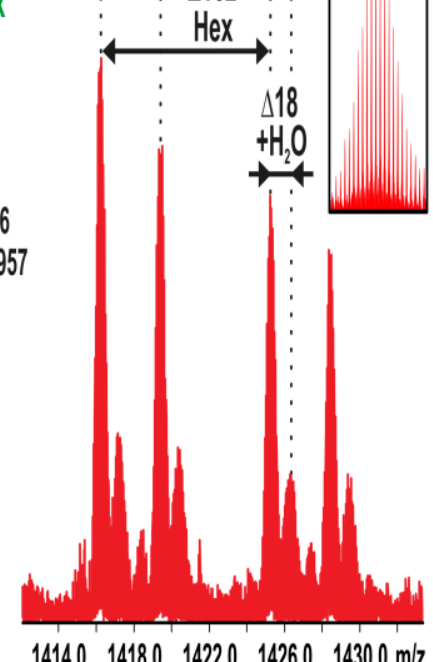

Figure 2 
(a)

\begin{tabular}{llllllllllllllllllllllllllllllllllllllllllll}
\hline 0 & $N$ & $N$
\end{tabular}

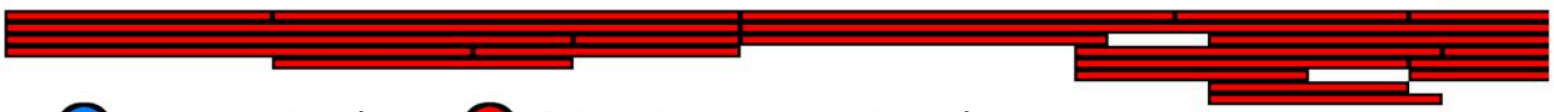

iPep, $100 \mu \mathrm{l} / \mathrm{min} \bigcirc$ irNep-1 + irRpn, $200 \mu \mathrm{l} / \mathrm{min}$

(b)

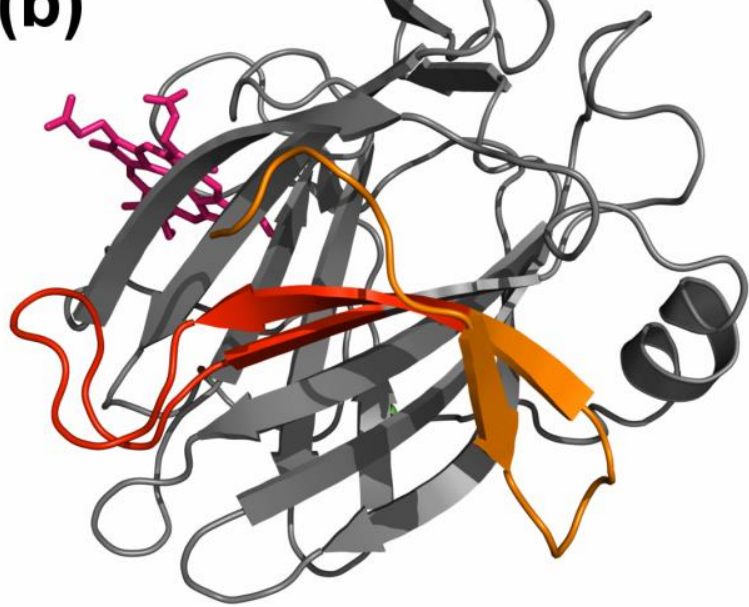

Figure 3 (c)

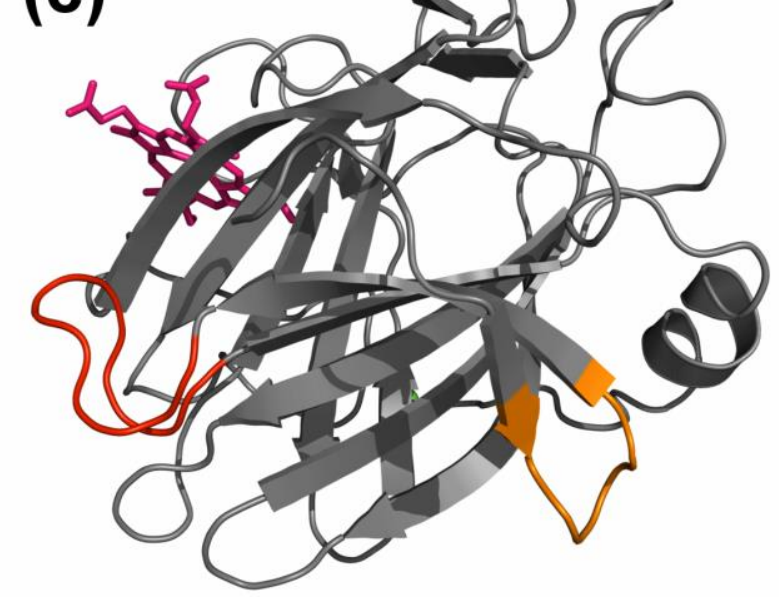


(a)
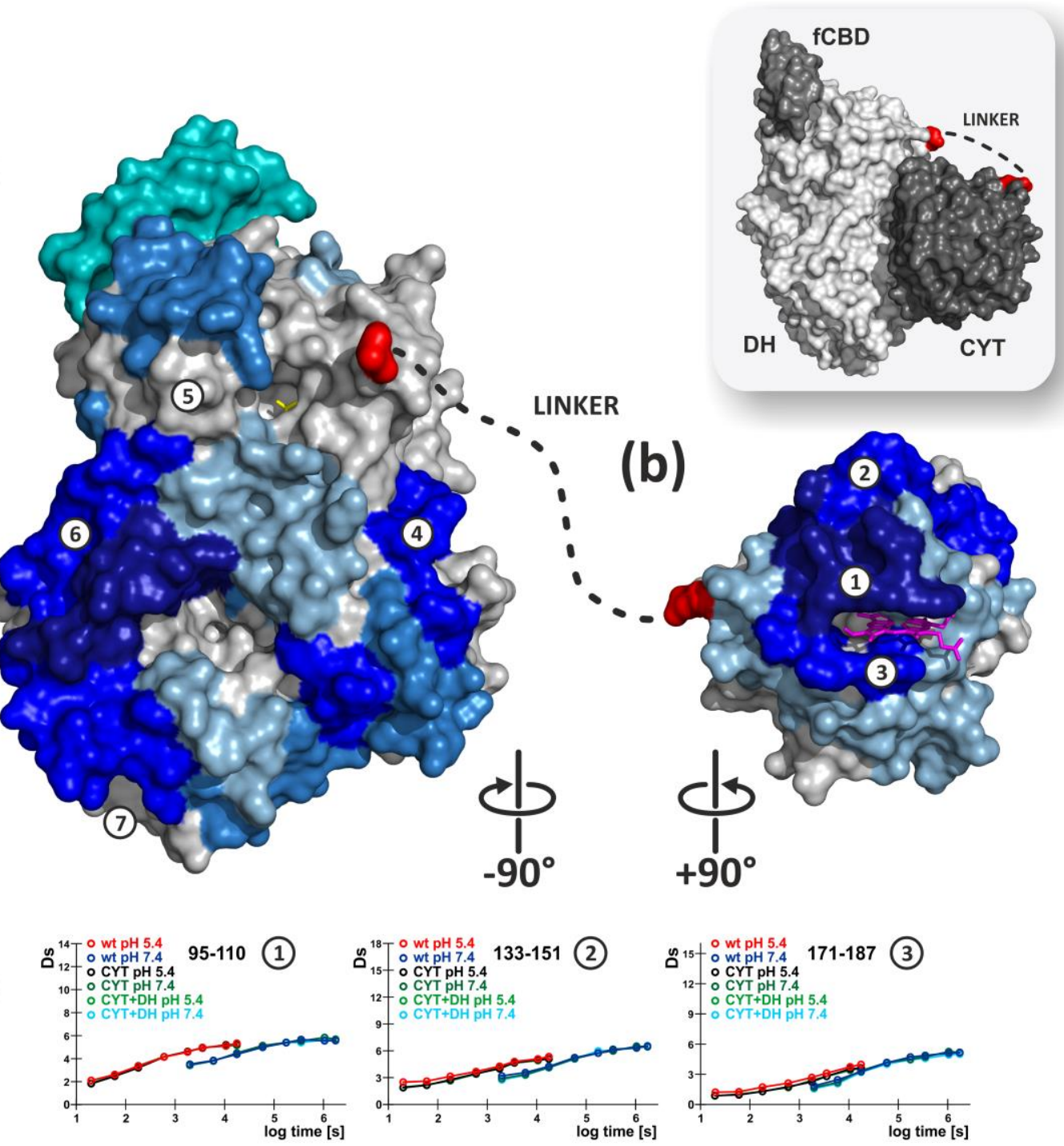

(b)
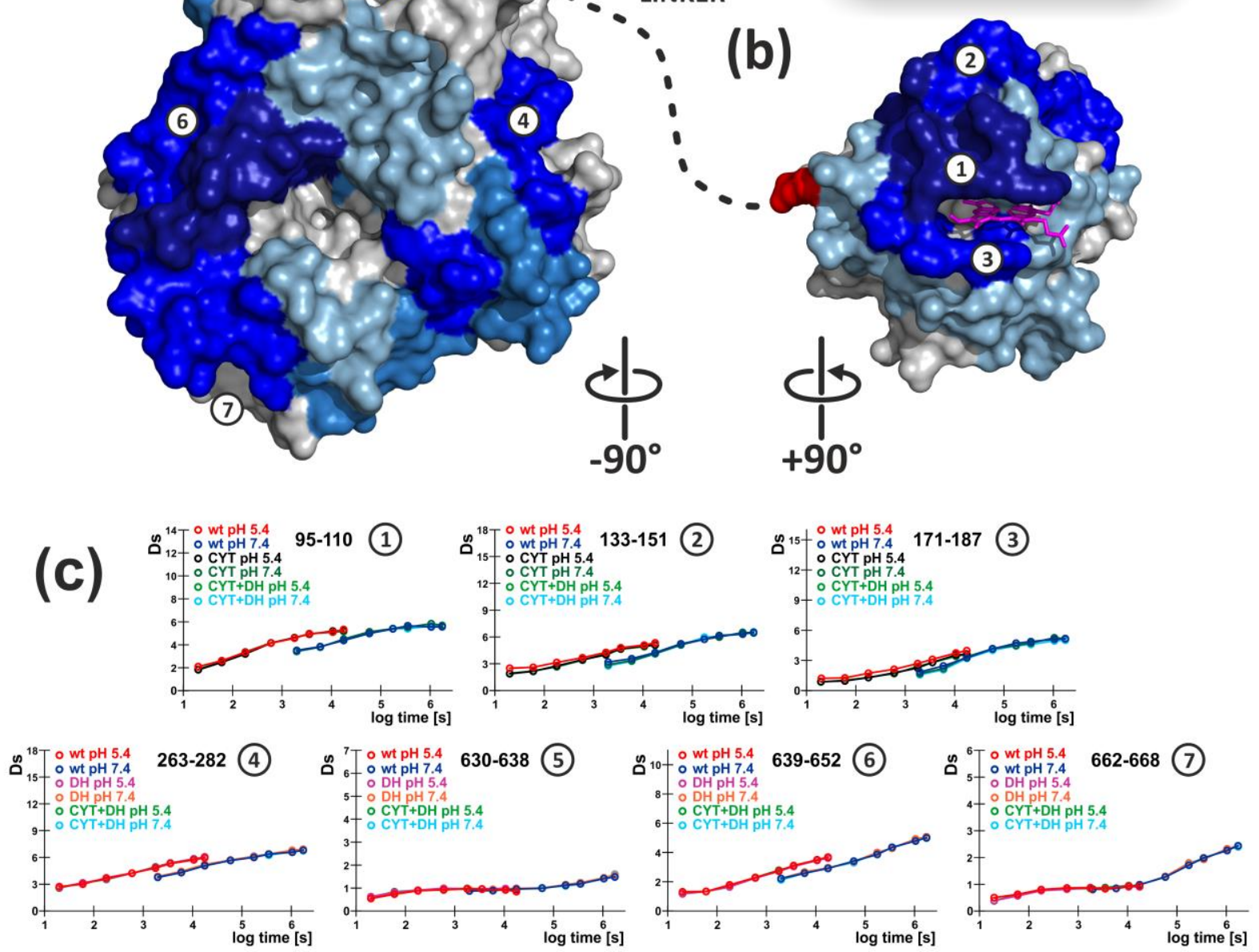

Figure 4 
(a)

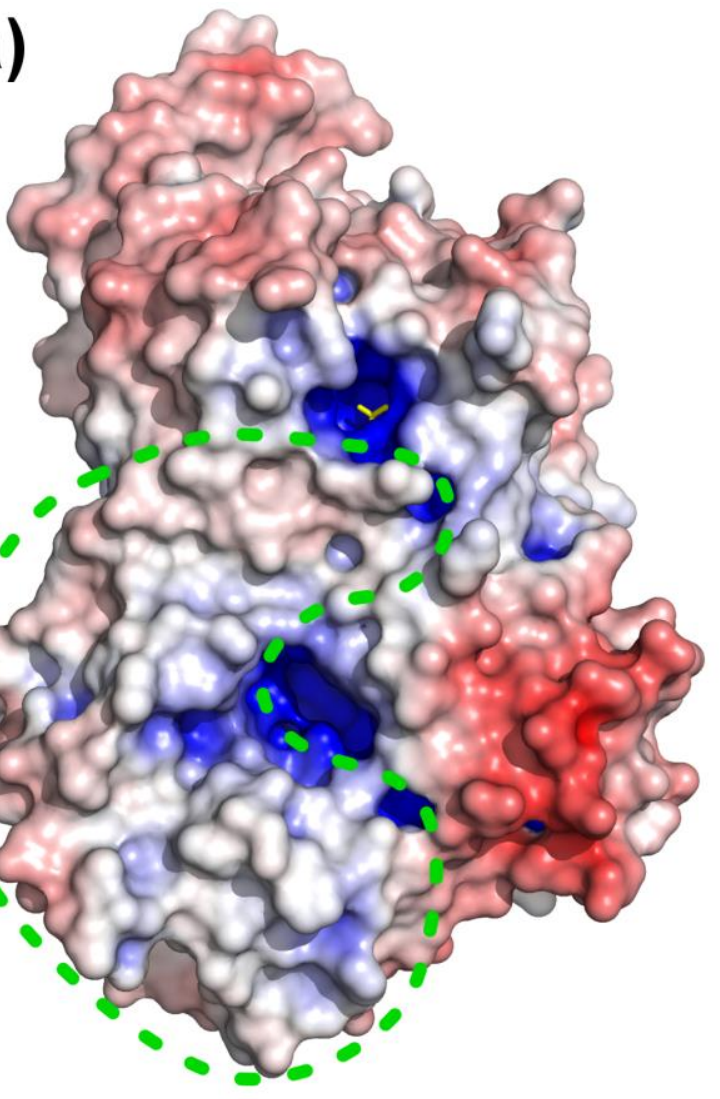

(c)

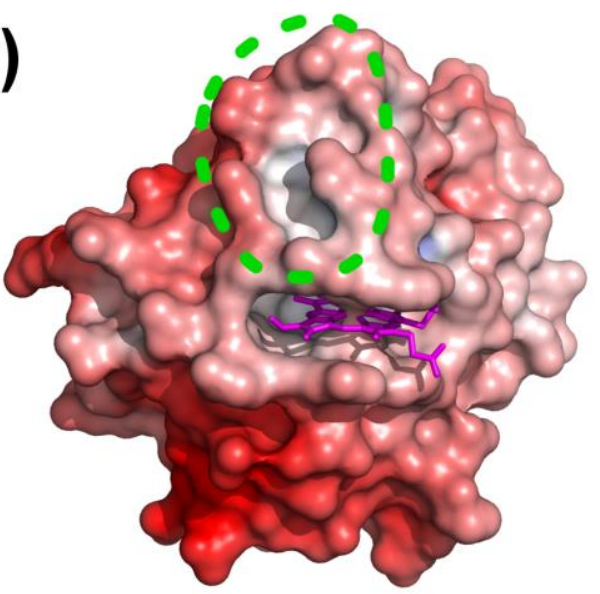

(b)

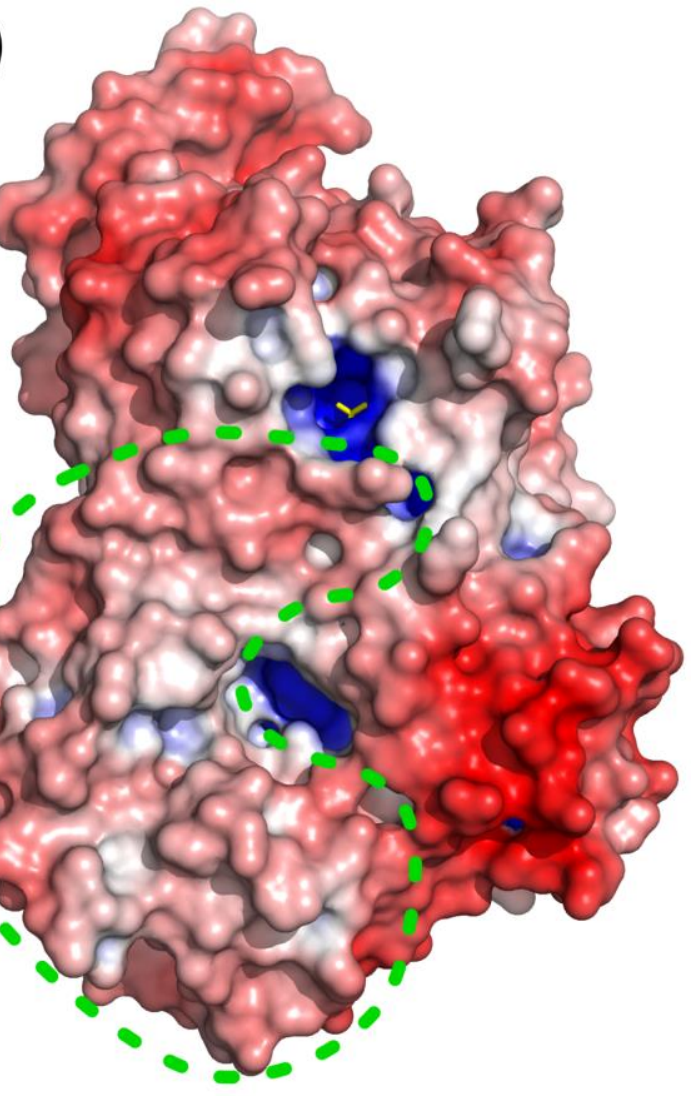

(d)

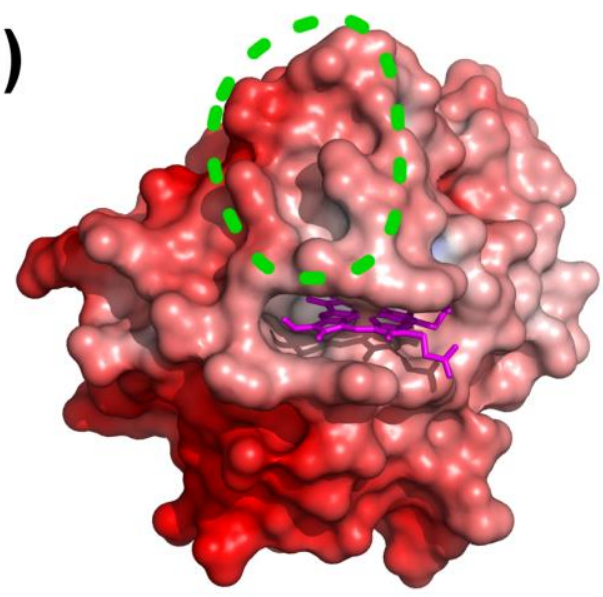

Figure 5 

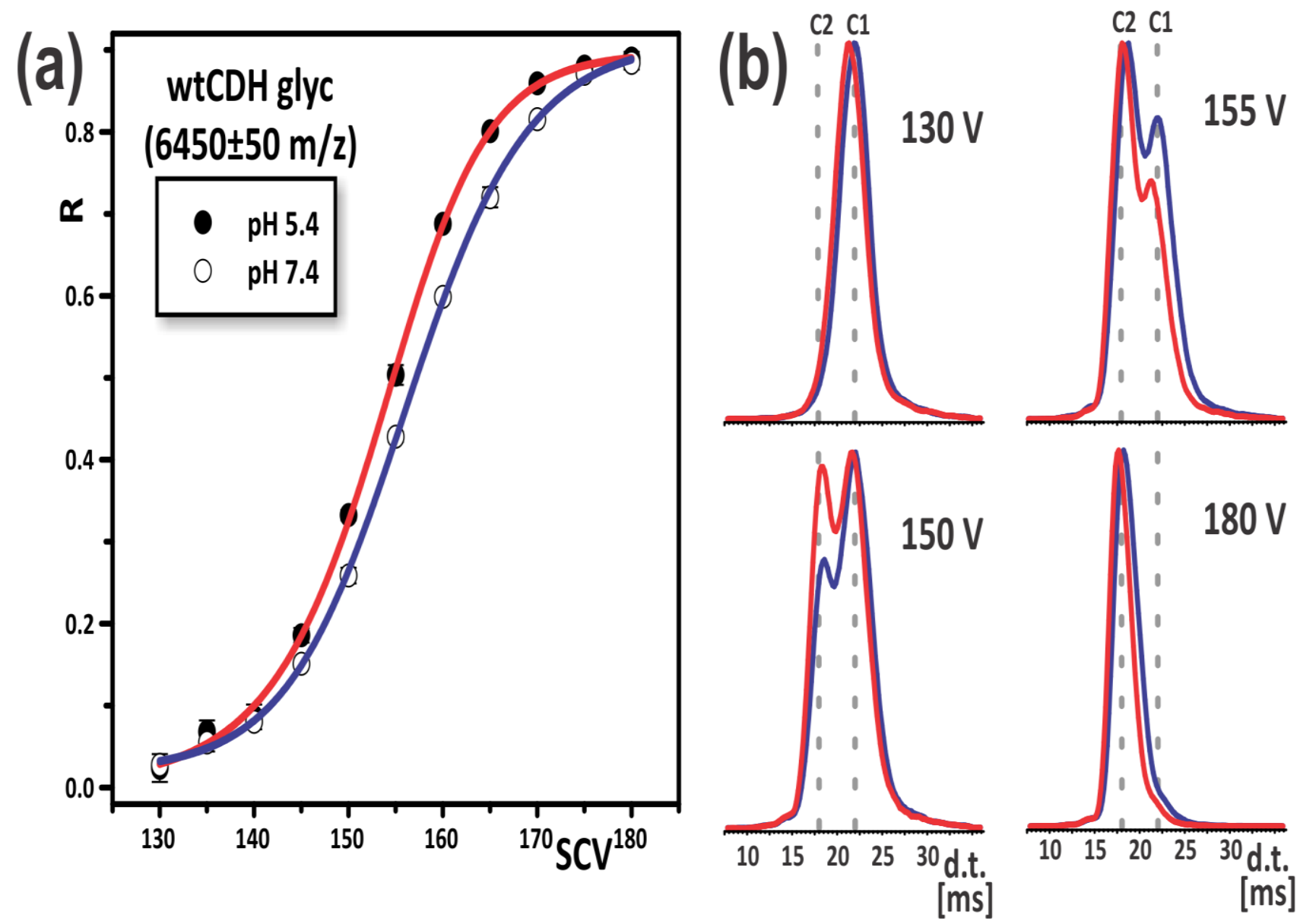

Figure 6 

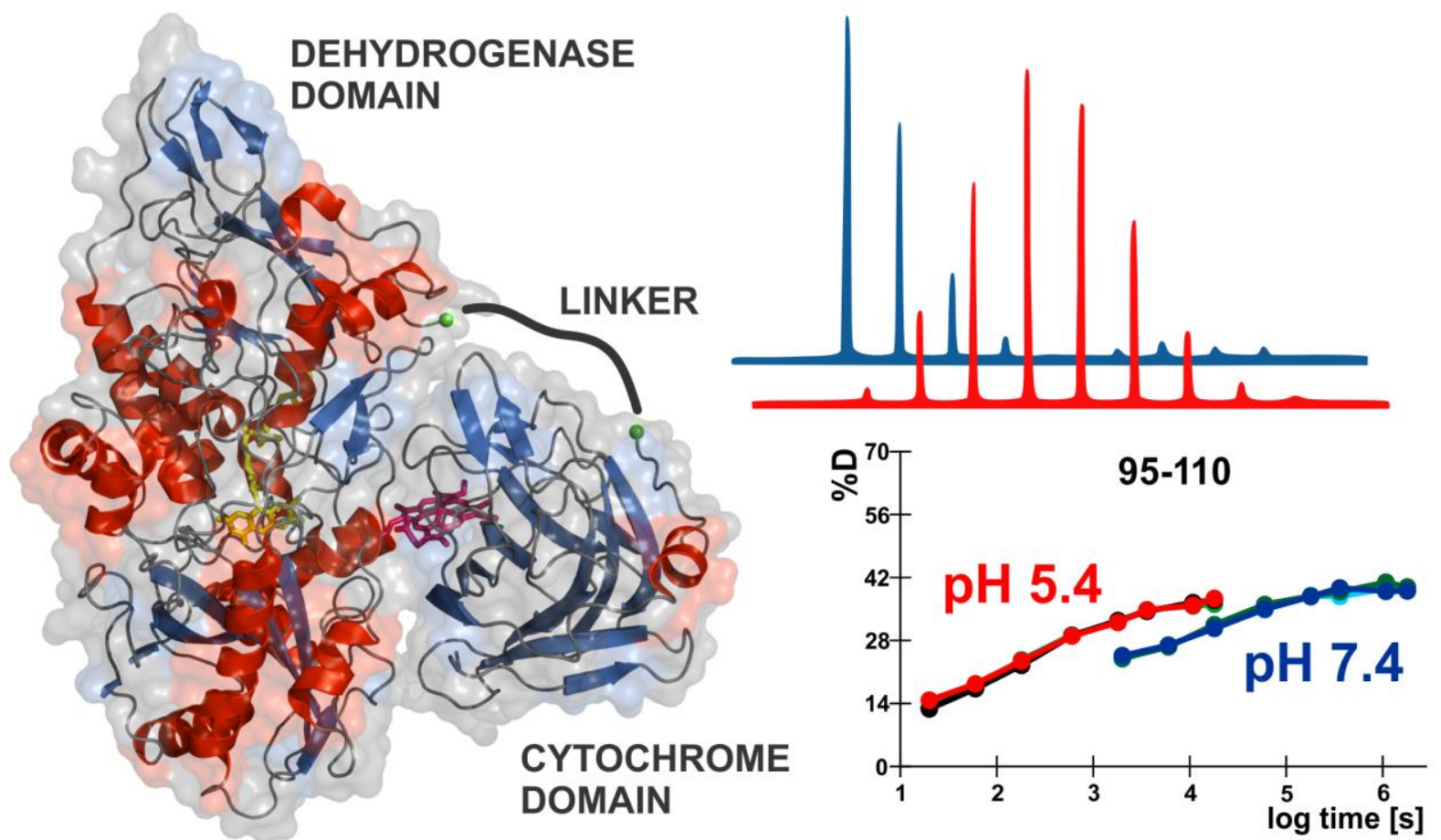

Graphical abstract 


\section{HIGHLIGHTS}

- Cellobiose dehydrogenase and its interdomain interaction was studied in solution

- $\mathrm{CDH}$ is extensively $\mathrm{O}$-glycosylated in the linker region

- $\mathrm{H} / \mathrm{D}$ exchange was used to describe structure changes in $\mathrm{CDH}$ at different $\mathrm{pH}$ values

- Ion mobility points at higher electrostatic repulsion between domains at neutral $\mathrm{pH}$

- Charge neutralization at acidic $\mathrm{pH}$ enables the domain interaction in $\mathrm{CDH}$ 